EOMmun Communication et organisation

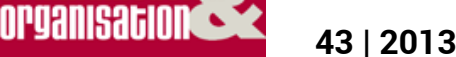

Réseaux sociaux entre médias et médiations

\title{
Essai philosophique sur les origines cybernétiques du coaching
}

Baptiste Rappin

\section{OpenEdition}

Journals

Édition électronique

URL : http://journals.openedition.org/communicationorganisation/4204

DOI : 10.4000/communicationorganisation.4204

ISSN : $1775-3546$

Éditeur

Presses universitaires de Bordeaux

Édition imprimée

Date de publication : 1 juin 2013

Pagination : 165-182

ISBN : 978-2-86781-883-7

ISSN : $1168-5549$

Référence électronique

Baptiste Rappin, «Essai philosophique sur les origines cybernétiques du coaching », Communication et organisation [En ligne], 43 | 2013, mis en ligne le 01 juin 2015, consulté le 10 décembre 2020. URL:

http://journals.openedition.org/communicationorganisation/4204; DOl : https://doi.org/10.4000/ communicationorganisation.4204 


\title{
Essai philosophique sur les origines cybernétiques du coaching
}

\author{
Boptiste RAPPIN
}

Peu nombreux sont les ouvrages de présentation générale sur le coaching. On trouve en effet dans la littérature sur cette pratique d'accompagnement les livres des praticiens, présentant le processus et les outils du coaching ; les essais critiques, plutôt issus du milieu universitaire (Brunel, 2004 ; Gori et Le Coz, 2006 ; Ebguy, 2008 ; Guilhaume, 2009) mais aussi des professionnels (Chavel, 2001; Fourès, 2004) ; les compilations, comme Le grand livre du coaching (Bournois, Chavel et Filleron, 2008) qui propose plus un tour d'horizon des pratiques et des approches qu'une réelle synthèse. Deux ouvrages font néanmoins exception dans ce paysage, par leur vocation même : le Que sais-je? d'Angel et Amar (2005) et le Topo de Brasseur (2009), qui se proposent chacun de présenter le coaching en 128 pages.

Toutefois, ni l'un ni l'autre ne proposent d'explorer la généalogie du coaching; ils font certes état de " champs théoriques » (Angel et Amar, 2005, Chapitre II) ou d' « ancrages théoriques » (Brasseur, 2009, Chapitre 2), mais les fondements philosophiques ne nous révèlent pas les origines historiques de cette forme spécifique d'accompagnement. C'est à cette carence de la littérature que notre article souhaite remédier. Ainsi la première partie présente tout d'abord une étude bibliométrique réalisée par Persson (2005) ; nous prenons alors appui sur les 20 références les plus utilisées par les coachs dans leurs ouvrages pour remonter vers le Nouvel Âge, l'école de Palo Alto et la cybernétique. Arrivé à ce dernier stade, nous procédons en seconde partie à la relecture du coaching par le cadre théorique de la cybernétique, relecture proposée au triple plan de l'ontologie, du politique et de l'éthique. La dernière partie souligne les apports de notre étude à la recherche académique sur le coaching.

\section{L’arbre généalogique du coaching}

Notre étude généalogique du coaching s'appuie sur un travail bibliométrique à partir duquel nous catégorisons les racines du coaching en trois pôles : le 
Nouvel Âge, l'école de Palo Alto et la cybernétique, confirmant ainsi les précédents travaux de Briffault et Champion (2005), Guilhaume (2010a) et Rappin (2011).

\section{Une étude bibliométrique comme point de départ}

Persson (2005) procède à une étude bibliométrique menée sur la littérature française du coaching parue entre les années 1992-2003, dans laquelle elle parvient à un classement des références générales utilisées par les coachs dans l'écriture de leurs ouvrages : cela nous permet de saisir les références mobilisées dans le contexte de l'émergence du coaching sur la scène française, et de comprendre a posteriori la trajectoire empruntée. Nous reproduisons ici les deux premières colonnes de son tableau pour les vingt premières places:

\begin{tabular}{|c|c|c|c|}
\hline RANG & RÉFÉRENCES & RANG & RÉFÉRENCES \\
\hline 1 & Socrate & 11 & Jung \\
\hline 2 & PNL & 12 & Palo Alto \\
\hline 3 & Maslow & 13 & Bible \\
\hline 4 & Rogers & 14 & Berne \\
\hline 5 & Freud & 15 & Bandler et Grinder \\
\hline 6 & AT & 16 & Taylor \\
\hline 7 & Erickson & 17 & Perls \\
\hline 8 & Watzlawick & 18 & Ulysse \\
\hline 9 & Gestalt & 19 & Descartes \\
\hline 10 & Bateson & 20 & Mentor \\
\hline
\end{tabular}

Tableau 1 : Les vingt première références classées par fréquence d'utilisation (Extrait de Persson [2005, p. 307])

Les références surlignées constituent le sujet d'exploration de cette première partie. Pourquoi avoir éliminé les autres ? Les premières renvoient à la stratégie d'argumentation des coachs qui tentent de légitimer leur pratique par des hauts patronages (pratique du storytelling [Persson et Rappin, 2010]) : il s'agit de Socrate, de la Bible, d'Ulysse, de Descartes, et enfin de Mentor. Les secondes (Freud et Jung), qui concernent la psychanalyse, peuvent certes servir de cadre théorique à certains coachs, mais n'en constituent pas pour autant des origines historiques du coaching. D'ailleurs, la plupart des praticiens se détournent explicitement de la psychanalyse, fondée sur un temps long et une orientation vers le passé, contrairement au coaching, centré sur le présent et le futur, qui se déroule dans un laps de temps réduit. Explorons alors les douze références restantes que nous surlignons en gras pour leur première apparition dans notre développement. 


\section{Le Nouvel Âge}

«Bien des idées et des pratiques du New Âge naîtront autour d'une ferme située à flanc de coteau entre San Francisco et Los Angeles, à Big Sur: dans le haut lieu californien d'Esalen. En cette Mecque mythique du Verseau convergent, à partir de 1961, des psychothérapeutes, des artistes, des scientifiques, des psychologues. Allan Watts, Arnold Toynbee, Carl Rogers, Paul Tillich, Carlos Castaneda, J. B. Rhine, Abraham Maslow, tous célèbres dans le monde des recherches sur la conscience et le cerveau, y séjournent. C'est la naissance du Mouvement du Développement du Potentiel humain » (Vernette, 1992, p. 48). L'Institut Esalen est une nébuleuse dans laquelle se forme un syncrétisme tout à fait impressionnant entre les pratiques spirituelles les plus iconoclastes (orientalisme, théosophie, spiritisme, chamanisme...) et les avancées scientifiques les plus récentes (la cybernétique) qui se rejoignent dans le moule du développement du potentiel humain. L'Institut véhicule une conception optimiste de l'homme, à l'image d'un Rogers : "Un des concepts les plus révolutionnaires qui soit sorti de notre expérience clinique est la reconnaissance accrue que le centre, la base la plus profonde de la nature de l'homme, les couches les plus intérieures de sa personnalité, le fond de sa nature animale, que tout ceci est naturellement positif, est fondamentalement socialisé, dirigé vers l'avant, rationnel et réaliste » (Rogers, 1998, p. 69). La relation d'aide se base donc sur la congruence, la non-directivité et l'empathie afin de laisser le potentiel de l'individu accompagné émerger.

En 1964, Fritz Perls s'installe en Californie et fréquente Esalen, et en devient même un des résidents permanents (De Vissher, 1991, p. 174). Abandonnant la pratique analytique au début des années 1950, il introduit une approche holiste dans la pratique thérapeutique : il s'agit de la Gestalt-therapie (Perls, 1963). Il fut en cela influencé par Wolfgang Köhler, Mollie Harrower, Kurt Lewin et Alexander Bavelas, sociologues et psychologues sociaux ayant pris part aux Conférences Macy (voir plus bas).

Bandler et Grinder vinrent observer les séances de Perls à l'Institut Esalen. Ils précisent eux-mêmes que la PNL résulte d' " un processus d'observation systématique de Virginia Satir, Milton H. Erickson, Fritz Perls et d'autres grands maîtres de la thérapie " (1982, p. 13). Satir faisait partie du Mental Research Institute de Palo Alto alors qu'Erikson assistait aux Conférences Macy et avait des liens avec le groupe «Bateson ». Notons pour conclure ce paragraphe que l'anthropologue, qui a inspiré les fondateurs de l'Institut, est venu lui-même passer quelque temps à Esalen, pour assister aux séances de Perls.

Eric Berne (2001) faisait lui aussi partie du public de Perls : l'AT fait partie du mouvement de développement de la personne. Le fondateur de l'AT a également été analysé par Erikson dans les années 1950 et tenait à la même période des séminaires en Californie : Carmel, Monterey, San Francisco et Palo Alto (De Visscher, 1991, pp188-191). 


\section{L'école de Palo Alto}

Cette rapide présentation du Nouvel Âge fait apparaître des connexions avec l'école de Palo Alto, notamment à travers les personnages de Bateson, d'Erikson et de Satir. Wittesaele et Garcia (1992) racontent avec force détails sa constitution et son histoire : retenons-en l'essentiel pour notre démonstration.

Le groupe «Bateson » prend forme dès 1953, sous la houlette de l'anthropologue éponyme, et réunit John Weakland, Jay Haley, William Fry et Don Jackson. Notons au passage qu'une fois épuisé le financement initial, le projet fut sauvé par les subventions de la Fondation Macy, du Fund for Psychiatry et du National Institute for Mental Health (trois institutions qui ont participé au financement des Conférences Macy). En 1959, le Mental Research Institute (MRI) est créé suite au désir de Don Jackson de développer le volet thérapeutique des réflexions engagées dans le « groupe Bateson ». Le MRI voit alors arriver deux thérapeutes (Jules Riskin et Virginia Satir) puis Paul Watzlawick en 1961 et sera, lui également, financé par le National Institute for Mental Health. Le groupe initial est constitué, s'enrichit de personnalités intellectuelles éclatées en différents lieux géographiques (on parle de « collège invisible »), et il en ressortira des apports théoriques et pratiques largement mobilisés dans le coaching : la nouvelle communication et les principes de la thérapie brève. N'oublions pas de mentionner l'influence d'Erik Erickson dans le "groupe Bateson ». Le psychiatre participa à plusieurs conférences Macy, et recevra surtout la visite régulière, à Phénix, de Jay Haley et John Weakland qui étudiaient la place des paradoxes dans la communication dans le cadre du "projet Bateson ». Ils seront les véritables diffuseurs de sa technique : hypnose, manière de faire changer les gens, façon de gérer la relation à travers l'écoute.

\section{La cybernétique et les conférences Macy}

Dès 1942, la Fondation Macy organise des conférences autour de ce qui se nommera plus tard la cybernétique ; elles réunissent pendant plus de dix ans des chercheurs prestigieux de différentes disciplines : mathématiciens, logiciens, anthropologues, psychologues, économistes... s'y retrouvent pour édifier une science générale du fonctionnement de l'esprit. Se constitue lors de ces conférences le réseau qui assurera la promotion et la postérité de l'approche cybernétique. Donnons quelques noms de ce cercle fondateur : le neuropsychiatre Warren McCulloch, le mathématicien Norbert Wiener, le neurophysiologiste Arthur Rosenblueth, le couple d'anthropologues Margaret Mead et Gregory Bateson, le psychologue Lawrence Franck, le psychanalyste Lawrence Kubie, le psychosociologue Kurt Lewin, l'informaticien Julian Bigelow, le physicien John von Neumann ou encore Heinz von Foerster ${ }^{2}$.

2 La liste complète comprenant l'ensemble des noms se trouve dans l'ouvrage de Dupuy (1999, p. 74). 
Pourquoi ces personnalités intellectuelles se regroupent-elles ? La cybernétique est en réalité un extraordinaire cadre unificateur qui permet de regrouper l'ensemble des sciences grâce à la notion d'information : "On voit apparaître dans les travaux de guerre de Wiener l'idée que l'information est un terme commun qui convient aux calculateurs mécaniques, aux réseaux de téléphone, aux transmissions radios, aux automates de tirs, et en définitive aux êtres humains qui sont imbriqués dans ces dispositifs " (Triclot, 2008, p. 72). Ce serait ainsi une erreur de ne pas prendre en compte la présence, initiale et absolument pas secondaire, de la cybernétique dans le champ des sciences humaines. Prenons le cas de Bateson qui reconnaît explicitement sa dette à l'égard de la cybernétique et de la théorie des systèmes dans l'avant-propos du premier tome de Vers une écologie de l'esprit (1977, p. 7-10). Yves Winkin (2001, p. 54 et suivantes) fait de lui l'un des fondateurs du « modèle orchestral » de la communication; en effet, l'anthropologue donne une extension illimitée à cette notion : la communication ne se limite désormais plus à la transmission d'un message, mais fait partie de tout acte et de tout évènement. Dans son ouvrage de 1951 coécrit avec Ruesch, Communication : the social Matrix of Psychiatry, Bateson propose ainsi de déplacer l'objet de la psychiatrie vers l'étude des communications interactionnelles. Il signe par là-même l'arrêt de mort de la conception individualiste au profit d'un homme social pris dans les rets du contexte (ou de l'écosystème) et de ses codes; d'où son intérêt pour les types logiques de Russell et la théorie du double bind (Bateson, 1980, «Vers une théorie de la schizophrénie », p. 9 et suivantes).

\section{Synthèse}

Le tableau suivant offre une synthèse de nos développements ; il permet de percevoir la continuité qui va de la cybernétique au Nouvel Âge. Les douze noms ressortant de l'étude bibliométrique apparaissent ainsi dans leur logique historique :

\begin{tabular}{|l|c|c|c|}
\hline & $\begin{array}{c}\text { Cybernétique } \\
\text { (à partir de 1942) }\end{array}$ & $\begin{array}{c}\text { École de Palo Alto } \\
\text { (à partir de 1953) }\end{array}$ & $\begin{array}{c}\text { Nouvel Âge } \\
\text { (à partir de 1961) }\end{array}$ \\
\hline $\begin{array}{l}\text { Fondateurs et/ou } \\
\text { membres actifs }\end{array}$ & Bateson & $\begin{array}{c}\text { Bateson } \\
\text { Watzlawick }\end{array}$ & $\begin{array}{c}\text { Rogers } \\
\text { Maslow } \\
\text { Perls (Gestalt) }\end{array}$ \\
\hline $\begin{array}{l}\text { Membres } \\
\text { participants }\end{array}$ & Erikson & Erikson & $\begin{array}{c}\text { Bateson } \\
\text { Berne (AT) } \\
\text { Bandler et Grinder } \\
\text { (PNL) }\end{array}$ \\
\hline
\end{tabular}

Tableau 2 : synthèse historique

Bateson est un personnage clef de cette généalogie : il apparaît dans les trois pôles historiques que nous avons mis en évidence. Or, c'est dans le cadre des Conférences Macy et de sa rencontre avec la cybernétique qu'il élabora son 
édifice théorique définitif. Il nous faut donc revenir aux fondements premiers de la cybernétique pour mieux entrevoir la nature du coaching.

Cette généalogie permet de mieux situer le lieu d'altérité du coaching, et d'entamer un travail d'herméneutique au sens que le philosophe Hans Georg Gadamer (1996) donnait à ce terme dans son maître ouvrage Vérité et Méthode. En effet, le travail d'interprétation, s'il résulte de la projection d'un cadre de pensée (ce que Gadamer nomme des «préjugés » dans lesquels il voit des «leviers de compréhension »), s'origine en premier lieu dans l'objet lui-même : répondre à la question de l'essence du coaching ne procède donc pas de l'application aveugle d'un cadre de pensée préexistant à cette pratique d'accompagnement, mais bien plutôt de l'écoute attentive de la vérité qui s'en dégage. C'est précisément pourquoi le présent essai prend appui sur la généalogie cybernétique du coaching.

\section{Le coaching comme forme cybernétique d'accompagnement}

Il n'est pas question, dans cet article, de rentrer dans les détails de la cybernétique, et de prendre position parmi les différentes interprétations qui en sont faites. Nous nous concentrerons sur les ouvrages de Norbert Wiener, car le mathématicien est le premier à avoir baptisé la nouvelle science émergente " cybernétique » et à lui avoir consacré un ouvrage dès 1948, suivi d'un second en 1950. Nous discutons ci-dessous des perspectives ontologiques, politiques et éthiques de la cybernétique, et raccordons ces réflexions à la pratique qui nous intéresse : le coaching.

\section{Ontologie de l'information}

Le point de départ de Wiener est une interprétation pour le moins audacieuse de la seconde loi de la thermodynamique. Mathieu Triclot (2008, p. 217 et suivantes) montre comment la cybernétique se construit « au moyen d'une analogie entre l'information et (...) l'entropie » et que cette analogie, de formelle chez Shannon, deviendra réelle chez Wiener. Shannon propose en effet un raisonnement probabiliste : plus un message est probable, moins la quantité d'informations est élevée ; au contraire, plus un message est improbable et plus il est riche en informations. Le lien est mathématique et formel. Toutefois, Shannon constate que la formule obtenue est identique à celle de Boltzmann pour l'entropie de l'énergie ; c'est pourquoi il en viendra à utiliser le terme d' "entropie " pour désigner le contraire de l'information. Mais Wiener pousse l'analogie plus loin en l'inscrivant dans le réel lui-même : «La notion de quantité d'information se rattache très naturellement d'ellemême à une notion classique en mécanique statistique : celle d'entropie. Tout comme l'information est dans un système une mesure de son degré d'organisation, l'entropie est la mesure du degré de désorganisation d'un système. L'une est simplement l'inverse de l'autre " (Wiener, 1948, p. 18). Le mathématicien inscrit l'information dans le tissu même du réel, celle-ci 
devient une "propriété matérielle des systèmes matériels » (Triclot, 2008, p. 226). Aussi l'organisation, qu'elle soit indifféremment celle des systèmes non-vivants ou des systèmes vivants, non seulement résulte d'une mise en forme, d'une in-formation, mais encore se définit comme un dispositif de lutte contre l'entropie informationnelle d'un monde destiné à l'in-différence. Ce raisonnement amène Wiener à édifier une véritable " théologie de l'entropie " (Breton, 1997, p. 33) et à initier une réflexion sur le Bien et le Mal. L'entropie résulte de l'action du diable. Mais de quel diable ? Celui des gnostiques ou des manichéens qui le conçoivent un semeur de troubles? $\mathrm{Ou}$ celui de Saint Augustin qui revêt le visage de l'imperfection ? Wiener (1954, p. 34-35) s'oriente vers cette seconde option : l'imperfection étant une donnée de l'univers, connaissable en tant que loi, c'est-à-dire invariable, elle peut être combattue par l'effort de la science. Si la négativité, le Mal, émanaient de la volonté capricieuse d'un esprit maléfique, du Malin, alors l'humanité serait impuissante face au désordre engendré. Or, pour Wiener, l'action de l'homme, soutenue par le savoir scientifique, peut et doit faire reculer l'entropie naturelle et sociale. Comment? En organisant le monde par l'information. Concept auquel nous devons, avec Wiener, accorder une très large extension : "Information is a name for the content of what is exchanged with the outer world as we adjust to it, and make our adjustment felt upon it »(Wiener, 1954, p. 17). Définition, qui inclut le langage, le comportement, la gestuelle autant que n'importe quel objet susceptible d'être échangé, et à laquelle fait écho la célèbre sentence de Palo Alto : «On ne peut pas ne pas communiquer» (Watzlawick, Beavin et Jackson, 1972, p. 46).

Les développements précédents incitent à une redéfinition du coaching et de sa mission. Celui-ci n'est pas simplement l'accompagnement en vue du développement du potentiel et des compétences dans le cadre d'objectifs professionnels, commel'annoncent les ouvrages de praticiens et les associations de coaching. Ses racines cybernétiques font de lui un processus plus subtil fondé sur le lien entre information et organisation. Ainsi, le coaching est une forme d'accompagnement visant à lutter contre l'entropie organisationnelle par l'introduction d'éléments informationnels extérieurs spécifiques (le coach, sa méthodologie et ses outils). Il s'agit, sur le plan ontologique, d'un dispositif dont la finalité est la transformation de l'homme en être informationnel et la création, chez le coaché, d'une disponibilité informationnelle propre à «l'âge de l'accès ». En témoignent d'ailleurs les techniques utilisées : la PNL promet de révéler les «secrets de la communication » alors que l'AT se fonde sur le concept de «transaction ». Le coaching fait partie intégrante de cette métaphysique du code qui a pris le relais de la métaphysique de l'énergie : "Contrôle cybernétique, génération par les modèles, modulation différentielle, feed-back, question/ réponse, etc. : telle est la nouvelle configuration opérationnelle » (Baudrillard, 1976, p. 89). Non plus l'énergie, la force, le solide, la tension, la motivation, 
l'accumulation, le stock, la production, etc. : mais la circulation, l'information, le flux, le liquide, la mobilité, la connexion, le système, la rétroaction.

On peut alors, par analogie avec le raisonnement augustinien de Wiener, établir l'ontologie du coaching : agent de l'ordre organisationnel, détectant l'origine du désordre collectif dans l'imperfection du coaché et non pas dans le travail obscur et gnostique d'une force extérieure (que pourrait être la concurrence, par exemple), le coaching a alors pour vocation de contribuer au combat contre l'entropie et à la restauration de l'organisation comme système stabilisé et fluidifié d'informations. Cette définition cybernétique du coaching permet de comprendre l'implicite, ou même plus fortement, l'impensé, des demandes de coaching. Si la raison immédiate et superficielle en est la performance médiocre voire l'échec, le motif profond en est une volonté de lutte contre l'entropie.

\section{Politique du feedback}

Le mot « cybernétique " provient du grec kubernetes et désigne à l'origine le pilote d'un navire; en d'autres terme, la cybernétique est l'art de manier le gouvernail, c'est-à-dire de gouverner. Mais la cybernétique possède un mode propre de gouvernement : elle est en effet, selon l'expression de Wiener, « la science du contrôle et de la communication ». Science du contrôle par la communication et science de la communication par le contrôle, pourrionsnous dire. Comment s'opère ce contrôle ? Qu'implique-t-il ?

Wiener introduit la notion de feedback et en fait un processus qui permet le contrôle d'un système (mécanique, physiologique, social) en l'informant des résultats de son action : "This last function, as we have seen, is called feedback, the property of being able to adjust future conduct by past performance " (Wiener, 1954, p. 33). Paquette (1987, p. 8) précise alors que le feedback comporte trois sous-processus : l'information, l'évaluation et la ré-action. Tout d'abord, l'action déclenche en retour une nouvelle arrivée d'informations sur les effets provoqués; ensuite, le système concerné procède à l'évaluation de l'action initiée en envisageant l'écart entre les conséquences et le but fixé. Le feedback peut être négatif (interruption de l'action) ou positif (il y a un déséquilibre, il faut donc agir). Enfin, le système ajuste son comportement et réagit au retour informationnel. C'est pourquoi le mathématicien Louis Couffignal (1963, p. 23) définit la cybernétique comme «l'art de rendre l'action efficace ». Le déroulement d'un processus de coaching est similaire : à partir d'un diagnostic initial (par exemple à l'aide de tests psychologiques comme le MBTI), tracer le chemin à parcourir pour atteindre les objectifs fixés, tout en ajustant ou réorientant la direction en fonction des actions accomplies et des résultats obtenus. Mais le feedback se manifeste aussi dans la technique même du coach basée sur le questionnement, l'effet miroir et la reformulation. Il possède alors deux spécificités : d'une part, le feedback est ici infini, dans la mesure où il est toujours possible de reformuler ce que le coaché exprime ; 
d'autre part, c'est un feedback « autophage » car l'information donnée par le coach provient du coaché lui-même.

Par ailleurs, le feedback existe car le système est orienté vers une finalité. Dès 1943, Rosenblueth, Wiener et Bigelow étendent la notion de finalité à l'ensemble des systèmes ; elle n'est désormais plus l'apanage de la liberté humaine et peut être soumise aux raisonnements scientifiques mécanistes et déterministes : «Le fait d'être dirigé par un but, comme cela a été défini ici, est tout à fait indépendant de la causalité, initiale ou finale. La téléologie a été discréditée principalement parce qu'elle était définie comme impliquant une cause subséquente dans le temps à un effet donné. Cependant, quand cet aspect de la téléologie a été abandonné, la reconnaissance associée de l'importance du but a aussi malheureusement disparu. (...) Le comportement téléologique devient ainsi synonyme de comportement contrôlé par un feedback négatif ». La définition du feedback suppose donc qu'un but soit assigné au comportement, qu'il s'inscrive dans un projet ou une forme finalisée : «Le terme de feedback est (...) employé (...) pour signifier que le comportement d'un objet est contrôlé par la marge d'erreur à laquelle se situe à un moment donné en référence à un but relativement spécifique » (Rosenblueth, Wiener, et Bigelow, 1943). D'où la question : qui fixe le but ? Qui décide de la finalité ? L'établissement du contrat de coaching répond à cette question. Il met en jeu le prescripteur (représentant l'organisation : hiérarchie directe, direction des ressources humaines, direction générale), le coach et le futur coaché. C'est au moment de cette rencontre liminaire que se définissent les objectifs assignés au processus d'accompagnement, créant les contours d'un espace de surveillance et d'une physique du pouvoir (Pezet, 2007b). Ainsi, pour prendre l'image du thermostat, le prescripteur fixe la température à atteindre alors que coach et coaché sont responsables du processus d'atteinte de cette valeur. Au total, gouverner, pour le prescripteur et le coach, c'est bien maîtriser chacun à leur niveau la circulation de l'information, et vérifier la manière font elle fait système, avec ses effets de rétroaction.

\section{Éthique de l'ouverture}

Paquette (1987, p. 7) affirme que la finalité appartient en propre au système et qu'elle est déterminée par un centre de commande qui fixe le but, par exemple une valeur de référence pour le thermostat. Il souligne quelques pages plus loin (p. 11) que les finalités peuvent être hiérarchisées et qu'il peut exister des valeurs de références supérieures. Nous percevons alors la puissance illimitée du concept de système : il existe toujours un sous-système inférieur ou un sur-système supérieur, ou encore des systèmes parallèles imbriqués... de telle sorte qu'il existe une multiplicité de finalités qu'il devient, atteint un certain degré de complexité, impossible de classer. La cybernétique implique le relativisme dans ses postulats mêmes. Relativisme présent dans le coaching et qui transparaît dans un certain nombre de tensions relevées 
par Fatien (2009, p. 50-51). Par exemple, l'accompagnement est soumis à l'exigence de performance économique et managériale, qui se retranscrit dans la fixation des objectifs, mais il est également perméable à des normes sociales valorisant le bien-être. Il promeut ainsi à la fois une éthique libérale de la performance et une éthique « humaniste » de l'épanouissement personnel et du développement de soi.

Par ailleurs, le coaching véhicule des valeurs dites " humanistes ", quitte à parfois tomber dans l'angélisme (Rappin, 2005). Il hérite de l'optimisme de Rogers qui considérait la nature humaine comme fondamentalement bonne, sociable et rationnelle. Il promeut l'écoute, la tolérance, le respect, la compréhension, le dialogue, l'empathie, le non-jugement. Toutefois, ces valeurs prennent une autre dimension lorsqu'elles sont recontextualisées dans le cadre de la cybernétique et ramenées à la dimension fondamentale de l'ouverture. En effet, si, du point de vue ontologique, l'organisation résulte de la circulation de l'information, et si, du point de politique, le feedback permet de s'assurer du contrôle de cette circulation, alors il faut créer les conditions éthiques, c'est-à-dire effectives chez l'être humain, d'une telle circulation : à savoir faire de la circulation de l'information une valeur en soi susceptible d'être incarnée. Le coach est précisément le modèle de cette incarnation, lui chez qui la technique et l'axiologique se rejoignent pour se confondre (par exemple, le dialogue comme technique ET comme valeur). Le coaching mise donc sur l'imitation du coach par le coaché dont le couronnement éthique donnera lieu à une nouvelle figure du manager : le manager-coach. En conclusion, grâce aux techniques mobilisées déjà citées, le coaching part en croisade contre les managers fermés, in-disponibles, dé-connectés. Et le relativisme souligné plus haut de se résoudre : car l'ouverture devient la condition de possibilité de la performance (plus de flexibilité car à l'écoute de ses managers, collaborateurs et clients) et de l'épanouissement personnel (reposant sur l'écoute de soi et l'accueil non-jugeant des autres).

\section{Discussion et apports à la recherche}

La partie précédente a entrepris une relecture du coaching par le prisme de la cybernétique, lecture justifiée par la généalogie mise en exergue dans la première partie. Nous discutons à présent des apports de notre travail à la recherche académique sur le coaching, et reproduisons la structure tripartite précédente : ontologie, politique, éthique. De façon générale, notre apport consiste dans le déplacement des problématiques existantes ainsi que dans la mise en évidence de la caducité de certaines catégories analytiques employées.

\section{Ontologie : de l'accumulation à la circulation}

Le postmarxisme de la sociologie clinique propose une vision critique du coaching comme incarnation d'un nouvel être-au-monde caractérisée par la gestion de soi. Alors que le capitalisme industriel reposait sur le « compromis 
fordiste ", le capitalisme financier est à l'origine d'un " appareil psychique organisationnel " qui joue sur l'identification affective et devient source de plaisir et de souffrance pour le salarié (Pagès et al., 1998 ; Aubert et de Gaulejac, 1991). Reprenant les leitmotivs, notamment celui de la réification, de l'école de Francfort, de Gaulejac (2005, p. 57) dénonce l'idéologie du management et de la gestion fondée sur l'objectivité, le fonctionnalisme, l'expérimentalisme, l'utilitarisme et l'économisme.

Se produit ainsi une « extension du domaine du management » qui n'épargne rien de ce monde et en vient à concerner l'homme : pour preuve, cette notion de " capital humain ", forgée par l'économiste Gary Becker, qui tend à réduire l'homme au statut de capital à faire fructifier. Le coaching fait donc partie des ces investissements en ressources humaines qui valorisent « l'entreprise de soi ", c'est-à-dire une " comptabilité existentielle » (De Gaulejac, 2005, p. 150) dont les maîtres-mots sont : gestion de soi, gestion de son temps et de ses priorités, gestion de ses émotions, faire le bilan... Les outils et les raisonnements préalablement appliqués aux machines et aux investissements se déplacent vers les êtres humains réduits à n'être que des facteurs ou des ressources de l'entreprise. De même, Ebguy (2008, p. 65-67) évoque une «marchandisation de soi » et d'une soumission à l'ordre de la performance.

Cette argumentation, pour séduisante qu'elle soit, ne prend pas acte de la transition que nous vivons entre la sphère capitaliste de l'accumulation et la sphère informationnelle de la circulation, la seconde englobant désormais la première. Tout en considérant, d'un point de vue phénoménologique, les mutations du système technoéconomique, elle repose encore pourtant sur les catégories d'analyse et de critique du capitalisme industriel fondé sur l'accumulation. Le freudo-marxisme, pour être encore pertinent, doit procéder à une révolution conceptuelle : abandonner le point de vue énergétique sur l'inconscient au profit d'une informatique psychique ; ne plus considérer l'accumulation du capital comme l'Alpha et l'Omega du système, mais comme une fixité provisoire et instable dans le flux incessant caractéristique de l'âge de l'accès, mais comme un simple chapitre du grand récit utopique de l'information (Breton, 1997 ; Mattelart, 2009 ; Forest 2003). Marx (1976, p. 35) lui-même avait déjà ce trait de l'ère bourgeoise : "Ce qui distingue l'époque bourgeoise de toutes les autres, c'est le bouleversement incessant de la production, l'ébranlement continuel de toutes les institutions sociales, bref la permanence de l'instabilité et du mouvement ». Ainsi le coaching ne doit-il plus, nous semble-t-il, se penser en termes de capitalisation et de stockage mais en ceux de fluidification et de circulation. Il est moins une accumulation de connaissances de soi et une acquisition de compétences qu'un apprentissage à rendre celles-ci liquides et mobiles, c'est-à-dire accessibles et disponibles (pour lui, pour ses collègues, pour l'organisation...). La congruence est là qui rapproche le coaching des pratiques de management propres au nouvel esprit 
du capitalisme (Boltanski et Chiapello, 1999) et de la vie liquide qui caractérise la seconde phase de la modernité (Bauman, 2006).

\section{Politique : du pouvoir et de la domination au contrôle et à la régulation}

Un second apport la perspective cybernétique est de reposer à nouveaux frais la question du politique et du gouvernement des hommes dans le coaching. S'inspirant des thèses de Foucault sur le pouvoir pastoral, Valérie Brunel (2004) voit dans les pratiques de développement personnel dans les entreprises une nouvelle forme de gouvernementalité qui repose sur la proximité bienveillante et la relation horizontale du pasteur et de son troupeau. Introduisant une modification substantielle des relations interpersonnelles et des rapports sociaux, le développement personnel, et le coaching à sa suite, opèrent une euphémisation des rapports de force au profit d'une gouvernementalité libérale qui s'appuie sur la servitude volontaire des individus. Guilhaume (2009, p. 127-132), quant à elle, présente le coaching comme étant au service d'une "domination plus subtile ». Nous l'observons : l'argumentation se déroule en termes de "pouvoir » et de «domination », renvoyant au paradigme énergétique. Or, précisément, la notion de control employée par Wiener ne se laisse pas saisir dans ce champ sémantique : comme le souligne Breton (1992/1997, p. 20), « là où le français met du «pouvoir» dans la notion de contrôle, l'américain y voit d'abord de la «régulation» et de la «commande» (...). Ainsi, pour Wiener, toute réflexion sur la communication est associée à l'idée de régulation, de commande et de maîtrise ».

Dans ce cadre-là, le coach est moins le vecteur d'un pouvoir pastoral que celui qui assure, par son action, le bon fonctionnement de l'organisation conçue comme pur système communicationnel. Il est en effet le vecteur de l'introduction de la commande, c'est-à-dire de la finalité organisationnelle, geste inaugurant le fonctionnement cybernétique d'autorégulation du salarié et de son contrôle par feedback par l'organisation. En tant que dispositif de circulation d'informations (entre le coach, le coaché et le prescripteur, entre le coaché et le coach, entre le coaché et le coaché lui-même) et d'accumulation de mémoire (cognitive et comportementale) en vue d'apprendre à corriger ses erreurs et à réduire les écarts par rapport à l'objectif final, le coaching ne se définit pas comme une nouvelle forme de gouvernementalité et d'exercice du pouvoir, mais plutôt comme un système de régulation des pensées et des actions humaines. De ce point de vue, il paraît plus judicieux de replacer cette « nouvelle forme de contrôle social » dans l'histoire de la normalisation, comme le font Gori et Le Coz (2006, p. 116-122), et de voir en cette pratique d'accompagnementun instrument de domestication. Domestication:processus d'assujettissement qui tue dans l'œuf toute tentative de construction ternaire de l'individu; nulle surprise, alors, que l'un des papes de la cybernétique et de la théorie des organisations, Herbert Simon (1983, p. 65), place le concept de 
« docilité » au cœur de l'apprentissage organisationnel : car seuls les individus dociles génèrent des " gains d'adaptation ».

La notion de "programme ", résultant de l'analogie entre cerveau et ordinateur, permet d'argumenter en ce sens. En effet, que devient "le système de régulation coaching " une fois le contrat arrivé à terme ? D'une part, l'organisation possède d'autres mécanismes de contrôle (" culture ", communication interne, entretiens d'évaluation...) ; d'autre part, l'objectif du coaching est de rendre le coaché autonome, c'est-à-dire capable d'apprendre à apprendre. En d'autres termes, de s'assurer que le coaché sera en mesure de traiter correctement l'information, de la décoder, en minimisant de lui-même les écarts entre output et goal. Il faut pour cela le programmer, la Programmation Neuro-Linguistique s'en étant fait une spécialité. Lisons, pour s'en convaincre, cette explication extraite d'un ouvrage classique :

« Programmation, parce que, tout au long de notre existence, nous nous programmons en mettant en place des façons de penser, de ressentir et de nous comporter que nous employons dans les multiples situations de notre vie. Si nous établissons l'analogie avec l'informatique, le matériel (hardware) est le même : nous avons tous un cerveau et un système nerveux. Ce qui change, ce sont les programmes (software) dont nous disposons pour nous servir de ce matériel. (...) Neuro, parce que cette capacité de nous programmer repose sur notre activité neurologique. (...) Dans notre pratique, nous nous demandons comment nos clients perçoivent leur environnement, quelles sont les parties de cet environnement qu'ils retiennent et celles qu'ils laissent de côté, quelles sont les représentations qu'ils ont d'eux-mêmes et de ce qui les entoure, ou encore comment est-ce qu'ils stockent les informations dans leur mémoire et comment ils accèdent à ces informations quand ils en ont besoin. Le cas échéant, nous les aidons à réorganiser ces processus afin qu'ils puissent mieux les mettre à profit. » (Cayrol et de Saint Paul, 2002, p. 25).

Veuillez nous pardonner la longueur de cette citation ; mais elle a ceci de précieux qu'elle met en évidence, dans l'une des pratiques les plus utilisées en coaching, le champ sémantique (programme, informatique, perception, information, mémoire, accès...) et les raisonnements issus de la cybernétique. Aussi l'autonomie tant promise par les coachs doit-elle s'envisager comme auto-régulation et auto-programmation : non pas comme capacité de poser ses propres lois, comme l'indique l'étymologie grecque, mais bien comme aptitude à s'ajuster et s'adapter à la finalité du système. Comme l'a bien vu Guilhaume (2010b), communication et auto-évaluation du coaché vont bien de pair.

\section{Éthique : Vers un souci de soi contemporain}

Le «souci de soi » foucaldien (Foucault, 1984, 2001) est la porte d'entrée par laquelle certains auteurs abordent la question de la subjectivation dans le coaching. C'est le cas de Pezet (2007a) qui aborde le management en 
général et le coaching en particulier dans le cadre des Foucaldian Studies. Il opère ainsi le rapprochement entre le coaching et les techniques de soi que Foucault relate dans le cadre de la Grèce ancienne (Pezet, 2007b). Or, comme le souligne Frédéric Gros (2007, p. 106) dans le même ouvrage, la comparaison entre les pratiques antiques de la subjectivation et le coaching comporte trois limites fondamentales : contrairement au souci de soi grec, le coaching puise dans le fonds scientifique de la psychologie, il s'inscrit dans un modèle de gestion (et non pas dans un modèle politique), et il contribue à une finalité qui lui est extérieure (la performance de l'organisation). En effet, le souci de soi est une "structuration historico-éthique » et les techniques de subjectivation ne sont pas indépendantes des régimes de véridiction propres à des époques particulières Gros (2002, p. 229). Si le coaching est bien un processus de subjectivation, une technique de soi, que nous apprennent ses racines cybernétiques de la spécificité de ce souci de soi contemporain ?

Tout d'abord, le coaching est bien une technique de soi, qui officie autant sur l' " âme " que sur le corps. Concernant le premier volet, on peut citer l'utilisation des tests psychologiques, les exercices de clarification des valeurs, des objectifs et des compétences, le dialogue avec le coach, mais également les techniques de visualisation ou encore les outils basés sur la créativité (Rappin, 2012). Pour le second aspect, citons les exercices de respiration et de concentration, les techniques de méditation, le travail sur la posture et la stature. Mais quelle est la finalité de ce " travail sur soi »? Quel est le point commun au bref inventaire que nous venons de dresser? Tous ces outils concourent, chacun à sa manière et avec son effet spécifique, à la constitution d'un sujet ouvert et disponible, débarrassé de ses blocages tant physiques que psychologiques, susceptible d'accueillir l'information autant que de l'émettre. Capable d'être à l'écoute de son corps et de sonder ses aspirations les plus profondes (la subjectivation comme rapport à soi), en mesure de communiquer avec autrui dans l'empathie et le non-jugement (la subjectivation comme rapport à autrui), le sujet qui se construit dans le coaching est un sujet d'information et de communication. Aussi sommes-nous en désaccord avec une interprétation du coaching qui en fait un vecteur de développement ou de renforcement du narcissisme (Amado, 2004), entraînant repli sur soi et fermeture. Au contraire, les racines cybernétiques du coaching indiquent que cette pratique d'accompagnement tend à rendre les sujets transparents (Rappin, 2006), ouverts et disponibles à l'interaction.

\section{Conclusion}

Le coaching puise ses sources dans le Nouvel Âge, l'école de Palo Alto et la cybernétique. Notre généalogie est toutefois incomplète : s’il est vrai qu'elle se fonde sur une étude bibliométrique, c'est-à-dire sur les ouvrages mêmes que les coachs lisent pour présenter le coaching, il reste à mettre au jour les chemins parcourus par le Nouvel Âge jusquau début des années 1990, 
date de naissance du coaching. Malgré cette faiblesse, qui est également une future piste de recherche, notre généalogie établit les sources du coaching dans la " matrice " cybernétique. Débute alors la relecture de la pratique de l'accompagnement par le prisme des catégories puisées dans les écrits de Wiener et d'autres auteurs présents aux fameuses Conférences Macy : information/organisation, contrôle, feedback, finalité. Il en ressort une tout autre interprétation du coaching de ce que la littérature académique pouvait donner à lire jusqu'à présent. Il semble en effet que l'analyse du coaching mobilisant les concepts de motivation, capital, pouvoir, domination, etc. qui relèvent d'un paradigme énergétique devient inopérante si l'on prend acte de l'ancrage de cette pratique dans la cybernétique, c'est-à-dire dans la sphère de la communication. C'est pourquoi nous avons proposé de (re)définir le coaching comme dispositif de lutte contre l'entropie organisationnelle, procédant par l'adjonction et le contrôle rétroactif d'informations, et assurant une subjectivation du coaché fondée sur l'ouverture et la disponibilité. Cette relecture permet d'envisager le déplacement des problématiques et des angles d'attaque actuellement présents dans la littérature académique, notamment dans le courant critique principalement issu de la sociologie clinique.

\section{BIBLIOGRAPHIE}

AMADO G., "Le coaching ou le retour de Narcisse ? ", Connexions, 81, 2004, p. 43-51. AUBERT N. , DE GAULEJAC V., Le coût de l'excellence, Paris, Seuil, 1991.

BANDLER R., GRINDER J., Les secrets de la communication, Montréal, Le Jour, 1982. BATESON G., Vers une écologie de l'esprit, tome 1, Paris, Seuil, 1977.

BATESON G., Vers une écologie de l'esprit, tome 2, Paris, Seuil, 1980.

BAUDRILLARD J., L'échange symbolique et la mort, Paris, Gallimard, 1976.

BAUMAN Z., La vie liquide, Rodez, La Rouergue/Chambon, 2006.

BERNE E., Analyse transactionnelle et psychothérapie, Paris, Payot, 1971-2001

BOLTANSKI L., CHIAPELLO E., Le nouvel esprit du capitalisme, Paris, Gallimard, 2009.

BOURNOIS F., CHAVEL T., FILLERON A., Le grand livre du coaching, Paris, Eyrolles, 2008.

BRASSEUR M., Le coaching en entreprise, Paris, Dunod, 2009.

BRETON P., L'utopie de la communication, Paris, La Découverte, 1992-1997.

BRIFFAULT X., CHAMPION F., « Le coaching, "bâtard" du potentiel humain pour l'individu transformable d'aujourd'hui ", in Le coaching, enjeux, paradoxes et perspectives, Communication E Organisation, 28, 2005, p. 35-52.

BRUNEL V., Les managers de l'âme, Paris, La Découverte, 2004.

CAYROL A. DE SAINT PAUL J., Derrière la magie. La programmation neuro-linguistique, Paris, InterEditions, 2002. 
CHAVEL T., Le coaching démystifié. Comment réenchanter le management ?, Paris, Demos, 2001.

COUFFIGNAL L., La cybernétique, Paris, PUF, 1963.

DE GAULEJAC V., La société malade de la gestion. Idéologie gestionnaire, pouvoir managérial et harcèlement social, Paris, Seuil, 2005.

DE VISSCHER P., Us, avatars et métamorphoses de la dynamique des groupes, Grenoble, Presses Universitaires de Grenoble, 1991.

DUPUY J.-P., Aux origines des sciences cognitives, Paris, La Découverte, 1994-1999.

EBGUY R., Je hais le développement personnel, Paris, Eyrolles, 2008.

FATIEN P. , "Intérêt organisationnel du coaching individuel ", Vie E̋ Sciences Économiques, 182, 2009, p. 39-56

FOREST D., « Du prophétisme techno-communicationnel à la théolo-technologie », Quaderni, 49, 2002-2003, p. 17-30

FOUCAULT M., Histoire de la sexualité III. Le souci de soi, Paris, Gallimard, 1984.

FOUCAULT M., L’herméneutique du sujet. Cours au Collège de France 1981-1982, Paris, Gallimard Seuil, 2001.

FOURÈS E., Petit traité des abus ordinaires. Un pavé dans la mare du coaching en France, Paris, Éditions d'Organisation, 2004.

GADAMER H.-G., Vérité et Méthode, Paris, Seuil, 1996.

GORI R., LE COZ P., L'empire des coachs. Une nouvelle forme de contrôle social, Paris, Albin Michel, 2006.

GROS F., "Sujet moral et soi éthique chez Foucault ", Archives de Philosophie, 65, 2002/2, p. 229-237

GROS F., "Le "souci de soi" chez Michel Foucault : tentative de comparaison avec le coaching contemporain ", in PEZET É. dir. (2007a), Management et conduite de soi. Enquête sur les ascèses de la performance, p. 99-107, Vuibert 2007.

GUILHAUME G., L'ère du coaching. Critique d'une violence euphémisée, Paris, Éditions Syllepse, 2009.

GUILHAUME G. (2010a), "Une approche communicationnelle du coaching ", Communication E Organisation, n³7, p. 115-124.

GUILHAUME G. (2010b), « Le coaching destiné aux managers-communicateurs : un dispositif de communication ou d'évaluation ? ", Communication E Organisation, 38, 2010, p. 121-131.

MARX K., Manifeste du parti communiste, Paris, Éditions sociales, 1848-1976.

MASLOW A., Vers une psychologie de l'être, Paris, Fayard, 1968-1972.

MASLOW A., L'accomplissement de soi, Paris, Eyrolles, 2004.

MATTELART A., Histoire de l'utopie planétaire. De la cité prophétique à la société globale, Paris, La Découverte, 1999-2009.

PAGÈS M., DE GAULEJAC V., BONETTI M., DESCENDRE D., L'emprise de l'organisation, Paris, Desclée de Brouwer, 1979-1998.

PAQUETTE G., « Feedback, rétroaction, rétroinformation, réponse... du pareil au même ", Communication et langages, 73, 1987, p. 5-18.

PERLS F., Manuel de Gestalt-thérapie, Paris, ESF Éditeur, 1973-2003. 
PERSSON S., Contribution à la connaissance du phénomène coaching en entreprise, Étude exploratoire des représentations au travers des discours de praticiens, Thèse de doctorat ès Sciences de Gestion soutenue à l'Université Nancy 2, 2005.

PERSSON S., RAPPIN B., "Quel cadre de lecture pour apprécier la littérature professionnelle des coachs? ", XXI ${ }^{\text {me }}$ Congrès de l'AGRH, Rennes / Saint Malo, 2010.

PEZET E. dir., (2007a), Management et conduite de soi. Enquête sur les ascèses de la performance, Paris, Vuibert.

PEZET E. (2007b), "Le coaching : souci de soi et pouvoir ", in Pezet E. dir. (2007a), Management et conduite de soi. Enquête sur les ascèses de la performance, p. 77-98, Paris, Vuibert.

RAPPIN B., Le réenchantement du coaching, Paris, L'Originel, 2005.

RAPPIN B., "Le coaching et la transparence intégrale ", Revue Internationale de Psychosociologie et de Gestion des Comportements Organisationnels, 27, 2006, p. 203-220.

RAPPIN B., " Le talent du coach. Le New Age et son influence sur le coaching comme nouveau mode de régulation de la déception sociale ", Revue Internationale de Psychosociologie et de Gestion des Comportements Organisationnels, 42, 2011, p. 157-169.

RAPPIN B., « Le sujet moderne dans le coaching. Une lecture à partir des travaux de Charles Taylor », Management et Avenir, 53, 2012, p. 183-201.

ROGERS C., Le développement de la personne, Paris, Dunod, 1968-1998.

ROSENBLUETH A., WIENER N., BIGELOW J., " Behavior, Purpose and Teleology ", Philosophy of Science, 10/1, 1943, p. 18-24

SIMON H., Reason in Human Affairs, Stanford Ca, Stanford University Press, 1983.

TRICLOT M., Le moment cybernétique. La constitution de la notion d'information, Seysell, Champ Vallon, 2008.

VERNETTE J., Le New Age, Paris, PUF, 1992.

WATZLAWICK P., BEAVIN J.H.,JACKSON D.D., Une logique de la communication, Paris, Seuil, 1972.

WIENER N., Cybernetics Or Control And Communication In The Animal And The Machine, Cambridge Mass., The Technology Press, 1948.

WIENER N., The Human Use Of Human Beings, New York, Da Capo Press, 1950-1954.

WINKIN Y., Anthropologie de la communication, Paris, Seuil, 1996-2001.

WITTEZAELE J.-J., GARCIA T., A la recherche de Palo Alto, Paris, Seuil, 1992.

Résumé : L'article procède à une étude généalogique du coaching; il décèle la présence du Nouvel Âge, de l'école de Palo Alto et, en fin de compte, de la cybernétique. Le coaching doit alors se redéfinir en fonction de ses origines historiques : il apparaît alors comme un dispositif cybernétique de régulation des hommes, considérés comme êtres informationnels, dans les organisations. La dernière partie propose ainsi de déplacer les problématiques actuelles de la recherche académique sur le coaching.

Mots-clés : Coaching, Généalogie, Cybernétique. 


\section{$C \& O n^{\circ} 43$}

Abstract : The article shows historical roots of coaching: New Age, Palo Alto school and cybernetics. Following this inquiry, it proposes to redefine coaching: it then appears as a cybernetic apparatus of people regulation. The last part draws conclusions and moves the current problematic of academic work on coaching.

Keywords : Coaching, Genealogy, Cybernetics. 